\section{Programmed cell death protein1 (PD1)-expression in the microenvironment of classical Hodgkin lymphoma at relapse after conventional chemotherapy and at relapse on anti-PD1 treatment}

We are grateful to Taylor et al. for their comments on our results to programmed cell death protein 1 (PD1) expression in sequential biopsies of classical Hodgkin lymphoma (cHL) patients with progressive disease under anti-PD1 treatment. Furthermore, we appreciate sharing their results to PD1- and PD ligand 1 (PDL1) expression in biopsies of a case series of cHL patients with relapse post conventional chemotherapy.

In their case series, Taylor et al. found no evidence of a shift in PD1 or PDL1 expression at relapse after chemotherapy in paired lymph node biopsies. These results are in line with the analyses of Schnitter et al. who examined PD1 expression in 16 and PDL1 expression in 13 paired biopsies at initial diagnosis and after first-line chemotherapy (Schnitter et al., manuscript in preparation). Both analyses thus support our hypothesis that the increase of PD1 ${ }^{+} \mathrm{T}$ cells in Hodgkin/Reed-Sternberg cell (HRSC) vicinity is associated with anti-PD1 treatment and is not a general feature of relapsing disease. In 4 of 9 analyzed patients (patient 2, 7, 8 and 9) with progressive disease of cHL, under anti-PD1 treatment, we could document a notable increase of $\mathrm{PD} 1^{+} \mathrm{T}$ cells in HRSC vicinity. ${ }^{1}$

Additional analyses are required to further characterize the described PD1 ${ }^{+} \mathrm{T}$ cells in HRSC vicinity. As already discussed by Taylor et al., PD1 expression is a widespread marker of T-cell activation, but might also indicate the presence of follicular helper T cells as well as the development of exhausted, hyperactivated T cells as possible mechanism of resistance to anti-PD1 treatment. ${ }^{2}$ Appropriate T-cell activation as the reason for documented increase of $\mathrm{PD}^{+} \mathrm{T}$ cells seems rather unlikely since all most recent biopsies were taken in relapse on anti-PD1 treatment. The latter hypothesis is supported by the observation that in 2 patients with progressive disease under anti-PD1 treatment and documented increase of PD1 $1^{+}$positive T cells (patients 7 and 8 ), a disease stabilization could be achieved by doubling the dose of the anti-PD1 antibody. ${ }^{1}$ However, as already mentioned by Taylor et al., the interpretation of our results in patient 8 is even more complicated since the histology of the most current relapse changed to a lymphocyte-rich (LR) sub- type of $\mathrm{cHL}$, and $\mathrm{LR} \mathrm{cHL}$ in fact differs from other subtypes of $\mathrm{cHL}$ in respect to $\mathrm{PD} 1$-positive cells within the tumor specimen.

A direct contact (rosette) of HRSC to PD1 strongly positive $T$ cells has been described as a characteristic feature of nodular lymphocyte predominant HL (nLPHL) and of LR cHL. The diagnosis of nLPHL could be excluded in the most current tumor biopsy of patient 8 as well as in the subsequent case series. Taylor et al. reported a significantly higher PD1 expression in their LR cHL cases compared to nodular sclerosis or mixed cellularity $\mathrm{cHL}$ cases. ${ }^{3}$ In order to further evaluate rosetting of HRSC by PD1 positive T cells as well as PD1 and PDL1 expression in LR cHL, we have analyzed 10 specimens of LR cHL obtained at diagnosis before any treatment by immunohistochemistry as previously described ${ }^{1}$ and by fluorescence multistaining. Furthermore, we analyzed the PDL1 gene copy number by fluorescence in situ hybridization (Zytovision, Bremerhaven, Germany). As shown in table 1 and illustrated in figure 1 , the majority of these LR cHL cases show rosetting PD1-positive T cells. HRSC lack PD-L1 expression and copy number alterations. These findings confirm the observation by Taylor et al. In contrast to the results of Roemer et al. in 2016, even in cases with detectable PDL1 polysomy or copy gain, no relevant PDL1 expression could be detected. ${ }^{4}$ More analyses are required to understand whether inverse correlation of PD-L1 on HRSC and PD1 on rosetting T cells is a feature restricted to the $\mathrm{LR}$ variant of $\mathrm{cHL}$.

However, the documented lack of PDL1 expression on HRSC in this case series of LR cHL and in the analyses of Sakakibara et al. in $2018^{5}$ stands in marked contrast to our observation that in patient 8 of our case series, $90 \%$ of HRSC of the most current tumor biopsy showed a strong PDL1 expression. This case of LR cHL might therefore be regarded as an atypical variant of this subtype which may have developed from mixed cellularity or nodular sclerosis subtype under the influence of immunomodulatory treatment.

We are aware of the limitations of our analysis, including the mentioned predominantly descriptive nature of our results, a limited analysis of markers due to scarcity of tissue in relapse, cross-tissue comparison and a heterogenous patient cohort. Taylor et al. listed the comparison of PD1/PDL1 expression across different cHL histological subtypes as an additional limitation of our analysis; we agree that assessment of possible dynamics in

Table 1. Analysis of PD1 and PDL1 expression in a case series of LR $\mathrm{cHL}$.

\begin{tabular}{|c|c|c|c|c|c|c|}
\hline Case number & Diagnosis & Age & Sex & PDL1 IHC & PDL1 FISH amplification & PD1 rosetts IHC \\
\hline 1 & lymphocyte-rich cHL & 21 & $\mathrm{~m}$ & - & normal & present \\
\hline 2 & lymphocyte-rich cHL & 58 & $\mathrm{~F}$ & +- & n.e. & present \\
\hline 3 & lymphocyte-rich cHL & 47 & $\mathrm{~m}$ & $+/-$ & polysomy & present \\
\hline 4 & lymphocyte-rich cHL & 65 & $\mathrm{~m}$ & - & n.e. & present \\
\hline 5 & lymphocyte-rich cHL & 20 & $\mathrm{f}$ & + & normal & Absent \\
\hline 6 & lymphocyte-rich cHL & 50 & $\mathrm{~m}$ & - & normal & present \\
\hline 7 & lymphocyte-rich cHL & 51 & $\mathrm{~m}$ & $+/-$ & normal & present \\
\hline 8 & lymphocyte-rich cHL & 81 & $\mathrm{~m}$ & - & copy gain & present \\
\hline 9 & lymphocyte-rich cHL & 56 & $\mathrm{~m}$ & - & n.e. & Absent \\
\hline 10 & lymphocyte-rich cHL & 42 & $\mathrm{~m}$ & - & polysomy & Present \\
\hline
\end{tabular}

cHL: classical Hodgkin Lymphoma; IHC: immunohistochemistry; FISH: fluorescence in situ hybridization; n.e.: not evaluable; +: majoriity of HRSC positive; +/-: partial expression in HRS; -: no expression in HRSC. 


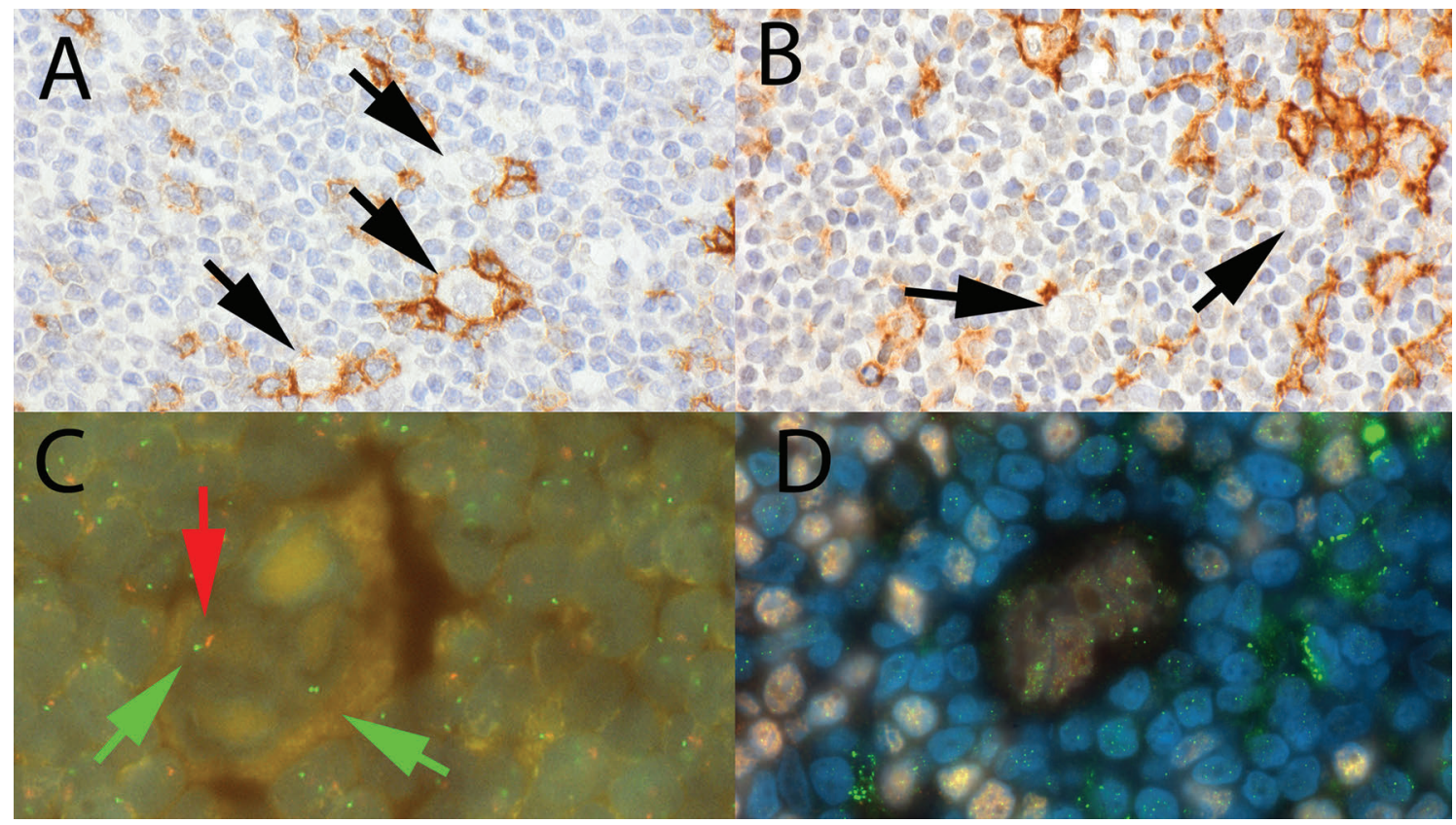

Figure 1. Immunohistochemical analysis and fluorescence in situ hybridization in a case of lymphocyte-rich classical Hodgkin lymphoma (case 8 from Table 1). A. Immunohistochemical staining for PD1 in case 8 (table 1) showing rosettes of PD1-positive T cells in vicinity of HRSC (arrows). B. Staining for PDL1 remains negative by conventional immunohistochemistry. C. Minor copy gain by fluorescence in situ hybridization with green arrows indicating the PDL1 probe and red arrows the centromeric control probe. D. Immunofluorescence multi-staining for Pax5 (red) to indicate HRSC and B cells and PDL1 (green). Blue colour represents DAPI nuclear staining. All images original magnification 1000x.

PD1/PDL1 expression in sequential biopsies might be considerably complicated by different histological subtypes. However, as indicated in retrospective analyses, change in cHL subtype in relapsed disease is frequent, and even more frequently, histopathological differentiation of the subtype is not possible. ${ }^{6}$ Thus, from our point of view, the analyzed tumor samples are representative for the examined patient cohort.

Despite the limitations listed, our results might contribute to the understanding of acquired resistance to anti-PD1 treatment in $\mathrm{cHL}$ and, as also concluded by Taylor et al., are therefore worth validating in a larger patient cohort. Furthermore, our data, the data presented by Taylor et al. as well as previously published data by Sakakibara et al. suggest that the LR subtype of cHL differs in PD1 and PDL1 biology from other subtypes of cHL.

Stephanie Sasse, ${ }^{1}$ Katharina Reddemann, ${ }^{2}$ Arjan Diepstra, ${ }^{3}$ Sven Borchmann, ${ }^{1,4,5}$ Ilske Oschlies, ${ }^{2}$ Antje Schnitter, ${ }^{2}$ Andreas Engert, ${ }^{1}$ Peter Borchmann ${ }^{1}$ and Wolfram Klapper ${ }^{2}$

${ }^{\prime}$ First Department of Internal Medicine and German Hodgkin Study Group, University Hospital of Cologne, Germany;

${ }^{2}$ Department of Pathology, Hematopathology Section, University Hospital Schleswig-Holstein, Christian-Albrechts-University, Kiel, Germany; ${ }^{3}$ Department of Pathology and Medical Biology,

University of Groningen, University Medical Centre Groningen, the Netherlands; ${ }^{4}$ Center for Molecular Medicine, University Hospital of Cologne, Germany; Else Kröner Forschungskolleg Cologne, University of Cologne, Germany
Correspondence: stephanie.sasse@uk-koeln.de or wklapper@path.uni-kiel.de

doi:10.3324/haematol.2018.207829

Information on authorship, contributions, and financial \& other disclosures was provided by the authors and is available with the online version of this article at www. haematologica.org.

\section{References}

1. Sasse S, Reddemann K, Diepstra A, et al. Programmed cell death protein-1 (PD-1)-expression in the microenvironment of classical Hodgkin lymphoma at relapse during anti-PD-1-treatment. Haematologica. 2019;104(1)e21-e24.

2. Jenkins RW, Barbie DA, Flaherty KT, et al. Mechanisms of resistance to immune checkpoint inhibitors. Br J Cancer. 2018;118(1):9-16.

3. Swerdlow SH, Campo E, Harris EL, et al., editors. WHO classification of tumors of haematopoietic and lymphoid tissues. 4th ed. Lyon: International Agency for Research on Cancer; 2017.

4. Roemer MG, Advani RH, Ligon AH, et al. PD-L1 and PD-L2 genetic alterations define classical Hodgkin lymphoma and predict outcome. J Clin Oncol. 2016;34(23):2690-2697.

5. Sakakibara A, Kohno K, Eladl AE, et al. Immunohistochemical assessment of the diagnostic utility of PD-L1: a preliminary analysis of anti-PD-L1 antibody (SP142) for lymphoproliferative diseases with tumour and non-malignant Hodgkin-Reed-Sternberg (HRS)-like cells. Histopathology. 2018;72(7):1156-1163.

6. Provencio M, Salas C, Milan I, et al. Late relapses in Hodgkin lymphoma: a clinical and immunohistochemistry study. Leuk Lymphoma. 2010;51(9):1686-1691. 